\title{
Internalizando la flexibilidad en la regulación del cambio climático: un análisis del caso brasileño
}

Christopher Kiessling

Recibido: 02/08/2020 ckiessling@conicet.gov.ar

CONICET - Universidad Católica de Córdoba, Argentina
Aceptado: $17 / 05 / 2021$
Publicado: 04/10/2021

\begin{abstract}
Resumen: A partir de la institucionalización del desarrollo sustentable en el contexto de la gobernanza global ambiental, los instrumentos económicos pasan a ganar legitimidad y percibirse como los mecanismos más eficientes para alcanzar la compatibilidad entre la promoción del desarrollo económico y la protección ambiental. En relación con la gobernanza global del cambio climático, este consenso se consolida con la firma del Protocolo de Kioto y los mecanismos de flexibilización diseñados para facilitar el cumplimiento de las metas previstas en el acuerdo. En este artículo se describe el proceso de localización de los mecanismos de flexibilización en el discurso público brasilero sobre cambio climático de 2005 a 2015 desde una perspectiva constructivista de las Relaciones Internacionales. El trabajo presenta tres aportes de relevancia en el marco de la literatura de internalización de normas internacionales ambientales y climáticas, a saber: el proceso de localización de los mecanismos de flexibilización en Brasil no ha sido lineal, el contexto de hegemonía discursiva del ambientalismo liberal fue un factor clave en la internalización de los mecanismos de flexibilización, y la sociedad civil así como otros actores subnacionales y no estatales son actores de una gran relevancia en la internalización e implementación de estas normas.
\end{abstract}

Palabras clave: Brasil; cambio climático; localización; Protocolo de Kioto; Mecanismo para el Desarrollo Limpio (MDL)

* Cómo citar este artículo: Kiessling, C. (2021). Internalizando la flexibilidad en la regulación del cambio climático: un análisis del caso brasileño. Relaciones Internacionales, 30(61), 121. https://doi.org/10.24215/23142766e121

Editor: Juan Alberto Rial, Instituto de Relaciones Internacionales Facultad de Ciencias Jurídicas y Sociales (Universidad Nacional de La Plata)
Entidad editora: Relaciones

Internacionales, es una publicación del Instituto de Relaciones Internacionales (Facultad de Ciencias Jurídicas y Sociales (Universidad Nacional de La Plata Argentina)

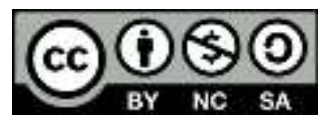

Reconocimiento-NoComercial Compartirlgual 4.0 Internacional (CC BY-NC-SA 4.0) 


\section{Internalizing flexibility on climate change regulations: An analysis of the Brazilian case}

\section{Christopher Kiessling ${ }^{1}$}

Abstract: Since the institutionalization of sustainable development within the context of global environmental governance, market-based environmental policy instruments began to gain legitimacy and to be perceived as the most efficient mechanisms to achieve compatibility between the promotion of economic growth and environmental protection. In relation to climate governance, this consensus was consolidated with the signing of the Kyoto Protocol and the flexibility mechanisms designed to facilitate compliance with the goals set forth in the agreement. This article describes the localization process of the flexibility mechanisms in the Brazilian public discourse on climate change from 2005 to 2015 from an International Relations constructivist perspective. The work presents three relevant contributions on the literature on the internalization of international environmental and climate norms, to wit: the process of localization of the flexibilization mechanisms in Brazil has not been linear; the context of discursive hegemony of liberal environmentalism was a critical factor in the internalization of flexibilization mechanisms; and civil society actors, as well as other sub-national and non-state actors, have great relevance in the internalization and implementation of these norms.

Key Words: Brazil; climate change; localization; Kyoto Protocol; Clean Development Mechanism (CDM)

\footnotetext{
${ }^{1}$ Doctor en Ciencias Sociales, FLACSO Argentina. Especialista en Derecho y Economía del Cambio Climático, FLACSO Argentina. Docente e investigador de la Universidad Católica de Córdoba, Becario postdoctoral del CONICET, cofinanciado por la Universidad Católica de Córdoba.
} 


\section{Introducción}

La gobernanza global ambiental se ha consolidado en estos últimos años como un tema de análisis de relevancia en las Relaciones Internacionales (RI), especialmente en el campo de la Economía Política Internacional (EPI).

Desde que los procesos socioecológicos son parte integral de los temas tratados por la EPI, los esfuerzos por integrar dentro una mirada analítica amplia la gobernanza ambiental en conjunto con el estudio de los procesos económicos y su regulación a escala internacional ha ido creciendo en estos últimos años (ver por ejemplo Saguier, 2018; Katz-Rosene \& Matthew, 2020).

Desde la celebración en 1972 de la Conferencia de Naciones Unidas sobre el Medio Ambiente Humano (CNUMAH), las normas de gobernanza global ambiental han tendido a converger gradualmente con normas económicas de carácter liberal propias del liberalismo incrustado (Ruggie, 1982). La institucionalización del concepto de desarrollo sustentable en la Cumbre de la Tierra de 1992 legitimó este proceso de convergencia hacia lo que Bernstein (2001) denomina ambientalismo liberal (AL). Este compromiso normativo postula la necesidad de abordar las políticas de protección ambiental y climática acompañadas de la promoción y el sostenimiento de un orden económico mundial liberal (Bernstein, 2001).

Así, la institucionalización del concepto de desarrollo sustentable en el marco del complejo normativo del AL tuvo dos consecuencias de suma relevancia. En primer lugar, legitima en el discurso internacional, la compatibilidad entre la búsqueda del crecimiento económico y la protección del medio ambiente (Comisión Mundial sobre el Medio Ambiente y el Desarrollo, 1987). En segundo lugar, los instrumentos económicos de mercado adquieren mayor legitimidad y pasan a ser percibidos como los mecanismos más eficientes para alcanzar la compatibilidad entre estos dos objetivos (Bernstein, 2001). De esta manera, el AL responde la pregunta de cómo abordar la regulación de los problemas ambientales, constituyéndose como guía para la gobernanza global del cambio climático².

Se consolida así una preferencia por la utilización de instrumentos económicos frente a los instrumentos regulatorios de comando y control, como las herramientas principales de política ambiental (Kiessling \& Pacheco Alonso, 2019).

Esta tendencia se institucionaliza en el marco de la CMNUCC, con la firma del Protocolo de Kioto (PK) y la definición de mecanismos de flexibilización como el Mecanismo para

\footnotetext{
${ }^{2}$ Dicha gobernanza, tiene en su centro a la Convención Marco de Naciones Unidas sobre el Cambio Climático (CMNUCC) adoptada en 1992.
} 
el Desarrollo Limpio (MDL) ${ }^{3}$, la Implementación Conjunta $(\mathrm{IC})^{4}$ y el Comercio de Emisiones $(\mathrm{CE})^{5}$. Estos mecanismos de flexibilización, fundamentalmente el MDL, adquirieron particular relevancia desde el año 2005 en el marco de la política climática de países del Sur Global, en especial Brasil, China e India. En un contexto de creciente interdependencia y de necesidad de aumentar la ambición en la acción climática, es sumamente relevante analizar los orígenes internacionales de las ideas que guían las políticas sobre cambio climático en países del Sur global y cómo ellas han sido adoptadas, reinterpretadas y plasmadas en la política de dichos países. En términos conceptuales, resulta necesario analizar los procesos de construcción de convergencia normativa entre la gobernanza global y los contextos domésticos nacionales a través de la localización de normas internacionales en el marco del ambientalismo liberal.

En este artículo se pretende realizar una contribución en este marco a partir del análisis de dicho proceso de localización (Acharya; 2004, 2011, 2012) y de los mecanismos de flexibilización, en el discurso público brasilero sobre cambio climático. Por discurso público brasilero se entiende el discurso sostenido por actores estatales y no estatales brasileros (actores gubernamentales, del sector empresarial, académicos, miembros de organizaciones no gubernamentales y movimientos sociales, periodistas, entre otros). La delimitación temporal del análisis abarca desde la entrada en vigor del Protocolo de Kioto en 2005 hasta la firma de un nuevo tratado, el Acuerdo de París, en 2015.

\section{Abordaje teórico-metodológico}

Desde una perspectiva constructivista de las Relaciones Internacionales, se ha desarrollado una profusa agenda de investigación que aborda la problemática de la internalización de normas ${ }^{6}$ internacionales en contextos nacionales (Finnemore \& Sikkink 1998; Risse, Ropp \& Sikkink 1999; Cortell \& Davis 2000; Krook \& True 2010; Niemann \& Schillinger 2016). Sin embargo, tal como la literatura contemporánea sobre la temática ha indicado (Niemann \& SchiIlinger, 2016; Kiessling, 2019a; 2019b), dichos modelos clásicos de internalización de normas

\footnotetext{
${ }^{3}$ EI MDL perseguía dos objetivos: I. Generar flujos de financiamiento y provisión de tecnologías y procesos de producción limpios, para asistir a los países miembros de la CMNUCC que no forman parte del Anexo I (países no industrializados y en vías de desarrollo) en la descarbonización de sus economías, y ll. Facilitar a los países pertenecientes al Anexo I (aquellos países desarrollados con un alto nivel de industrialización) el cumplimiento de sus metas de reducción de emisiones de gases de efecto invernadero (GEls) a través del financiamiento de proyectos de mitigación en economías en desarrollo donde resultan más eficientes en términos de costo-efectividad.

${ }^{4}$ La IC presentaba un funcionamiento semejante al MDL, con la diferencia que operaba exclusivamente entre países Anexo I.

${ }^{5}$ El sistema de comercio de emisiones era un sistema de limitación y comercio entre países Anexo I, en vinculación con el MDL y la IC. La clave del mecanismo era que los países que redujeran sus emisiones más de lo comprometido, podrían vender los certificados de emisiones excedentes a los países que no hubieran cumplido con su compromiso.

${ }^{6}$ Las normas han sido definidas tradicionalmente como estándares de comportamiento apropiado para actores con una identidad dada (Katzenstein, 1996).
} 
poseen ciertas limitaciones para comprender tanto los recorridos no lineales de internalización como los procesos de contestación de las normas (Wiener 2009; Wolff \& Zimmermann 2016). Frente a estos desafíos, se ha formulado el concepto de convergencia normativa, donde el proceso de difusión de normas no se comprende como una transferencia directa del contenido de la norma internacional a la esfera doméstica, ni como un proceso lineal, sino más bien como un proceso dinámico e impredecible que oscila entre percepciones de congruencia e incongruencia entre las normas globales y las condiciones domésticas (Stevenson, 2013). Así, la construcción de congruencia normativa puede potencialmente adoptar diferentes formas e incorporar un rango de diferentes actores locales estatales y no estatales. Dichos actores pueden (consciente o inconscientemente) impulsar procesos de cambio basados en su inconformidad con la percepción de incongruencia (o congruencia), en relación con las normas internacionales. Reconocer la dimensión discursiva de las normas permite cuestionar el supuesto de que estas últimas mantienen su esencia y significado inalterados durante el proceso de internalización. De hecho, la propia integridad de una norma internacional puede ser cuestionada incluso después de su aceptación retórica (Stevenson, 2013).

En este contexto, Acharya $(2004,2011,2012)$ propone el concepto de localización para referirse a la construcción activa, a través del discurso, de ideas ajenas por actores locales, que resulta en el desarrollo de una significativa congruencia entre las normas internacionales y creencias y prácticas locales (Acharya, 2012). En este marco, los procesos de localización incluyen dos mecanismos, denominados grafting ${ }^{7}$ y framing ${ }^{8}$. Ambos conceptos habilitan un proceso tendiente a seleccionar ciertos aspectos de una realidad percibida y dotarlos de una mayor prominencia en un discurso, de manera que promuevan determinada definición de un problema, su interpretación causal, evaluación moral y/o recomendación para su tratamiento (Stevenson, 2013). La localización puede comenzar con una reinterpretación y "re-representación" de la norma externa, pero tiene el potencial de extenderse a procesos más complejos de reconstitución normativa, para permitir la transformación de una norma externa de forma congruente con un orden normativo local preexistente (Kiessling, 2019a). Se trata, así, de un proceso en el que el papel de los actores locales se torna más significativo que el de los externos. En ese sentido, la localización es el principal dispositivo que permite la construcción y reconstrucción de la congruencia normativa entre normas internacionales y contextos internos (Kiessling, 2019a).

Asimismo, desde una perspectiva constructivista se han realizado contribuciones sustantivas en el campo de la EPI. Más allá de aportar un acercamiento reflexivo al pensamiento convencional basado en el reconocimiento de la naturaleza socialmente construida de las

\footnotetext{
${ }^{7}$ Grafting es una táctica empleada para institucionalizar una nueva norma al asociarla con una norma doméstica preexistente en la misma área temática que establece un mandato similar o comparable a la norma internacional (Acharya, 2004).

${ }^{8}$ Framing refiere al proceso activo de construcción de vínculos entre las normas existentes y las normas emergentes. A través del framing, los defensores de las normas internacionales en el contexto doméstico destacan determinados problemas mediante el uso de un lenguaje que los nombra, interpreta y dramatiza (Acharya, 2004).
} 
instituciones económicas, desde el constructivismo se ha propuesto un análisis de la EPI basado en enfatizar el carácter contingente de instituciones, prácticas y normas que condicionan el funcionamiento de la economía global (Widmaier, 2003; Abdelal, 2009; Abdelal, Blyth, \& Parsons, 2010; e.g). En particular, desde una aproximación constructivista de la EPI se han desarrollado investigaciones que abordan el estudio de la economía política del cambio climático, basadas en el argumento del valor normativo del ambientalismo (La Riviere, 2014), entre las cuales la conceptualización sobre AL tiene una centralidad indudable y a las que este trabajo pretende realizar una contribución.

Resulta importante en este punto profundizar ciertas referencias conceptuales sobre el AL, a modo de presentar las anticipaciones de sentido que guiaron esta investigación. Tal como se puede inferir del análisis anterior, el AL se caracteriza por aceptar prácticas como la liberalización del comercio y de las finanzas como un programa coherente con la protección ambiental a nivel internacional e incluso necesario para alcanzarla (Kiessling \& Pacheco Alonso, 2019). La legitimización de este compromiso normativo como guía para abordar el cambio climático configura el modelo a partir del cual organizaciones internacionales, Estados y otros actores no estatales interpretan su rol en la lucha contra el cambio climático, tanto a nivel local como a nivel regional e internacional.

A partir de diferenciar entre aquellas normas que (i) regulan aspectos relacionados con la responsabilidad y soberanía de los Estados, (ii) apuntan a la economía política del medio ambiente y el desarrollo y (iii) refieren a la gestión de los problemas ambientales, Bernstein (2001) identifica el siguiente complejo normativo emergente de la Declaración de Río sobre Medio Ambiente y Desarrollo (1992):

\section{Ambientalismo liberal como complejo normativo}

\begin{tabular}{|l|l|}
\hline $\begin{array}{l}\text { Responsabilidad y } \\
\text { soberanía de los } \\
\text { Estados }\end{array}$ & $\begin{array}{l}\text { 1. Soberanía sobre los recursos y protección del medio } \\
\text { ambiente dentro de las fronteras estatales. Existe } \\
\text { responsabilidad por la contaminación más allá de las fronteras } \\
\text { estatales } \text { (principios 2, 13 y 14). }\end{array}$ \\
\hline $\begin{array}{l}\text { Economía política del } \\
\text { medio ambiente y el }\end{array}$ & $\begin{array}{l}\text { 2. Responsabilidades comunes pero diferenciadas entre los } \\
\text { países desarrollados y los países en vías de desarrollo (Principios } \\
\text { 3,7 y 11). El desarrollo tiene prioridad si los costos de protección } \\
\text { del medio ambiente son demasiado altos (Principio 11). }\end{array}$ \\
\cline { 2 - 2 } & $\begin{array}{l}\text { 3. Libre comercio y mercados libres. La protección del medio } \\
\text { ambiente es compatible con la existencia de mercados libres } \\
\text { (Principio 12). }\end{array}$ \\
\hline
\end{tabular}

\footnotetext{
${ }^{9}$ Esta norma es anterior a la Declaración de Río, dicho documento establece dos ligeras modificaciones a dicha norma: a) Sería necesaria la notificación previa del potencial daño ambiental (Principios 18 y 19 de la Declaración de Río); b) Los Estados tienen derecho a explotar los recursos naturales para promover el desarrollo además de ser responsables de las políticas de medio ambiente (Bernstein, 2001).
} 


\begin{tabular}{|l|l|}
\hline \multirow{1}{*}{} & $\begin{array}{l}\text { 4. Transferencias internacionales libradas principalmente a los } \\
\text { mecanismos de mercado, excepto para los países menos } \\
\text { adelantados. }\end{array}$ \\
\cline { 2 - 2 } & $\begin{array}{l}\text { 5. Cooperación multilateral para el crecimiento económico y el } \\
\text { desarrollo centrado en las personas como prerrequisito necesario } \\
\text { para la consecución de otros objetivos (Principios 1, 7 y 27). }\end{array}$ \\
\hline $\begin{array}{l}\text { Gestión de los } \\
\text { problemas } \\
\text { ambientales }\end{array}$ & $\begin{array}{l}\text { 6. Mecanismos de mercado favorecidos por sobre los } \\
\text { instrumentos regulatorios. Principios de Precaución y } \\
\text { Contaminador-pagador institucionalizados (Principios } 15 \text { y 16). }\end{array}$ \\
\hline $\begin{array}{l}\text { Complejo normativo } \\
\text { resultante }\end{array}$ & AMBIENTALISMO LIBERAL \\
\hline
\end{tabular}

Elaboración propia a partir de Bernstein (2001).

Por su parte, resulta importante revisar los principios que, dentro del AL, abordan la gestión de los problemas ambientales; esto es, se preguntan cómo regular el cambio climático a escala internacional por medio de instrumentos económicos ${ }^{10}$. En concreto, en la gobernanza climática global todos estos instrumentos operan a partir de los mismos principios básicos: (i) la asignación de derechos de propiedad a las emisiones a través de diferentes certificaciones, (ii) la persecución de reducción de emisiones dónde resulte más eficiente, debido a que el impacto en la atmósfera producto de esta reducción es el mismo independientemente de dónde ésta se realice, y (iii) la creación de un mercado ${ }^{11}$ que permite transferir dichos créditos.

Atendiendo a estas consideraciones, el diseño metodológico propuesto para abordar la problemática analizada consiste en un estudio de caso a partir de una estrategia de rastreo de procesos (process tracing) de corte interpretativo (Bennett, 2010; Collier, 2011). Así, la anticipación de sentido que guía esta investigación reconoce un proceso de localización de los mecanismos de flexibilización que puede ser rastreado empíricamente a partir de plantear hipótesis sobre una serie de pasos que, de ser observados, se podrían reconocer como indicadores de la internalización de las normas referidas, en el período 2005-2015 en Brasil.

Asimismo, para rastrear empíricamente la internalización de los mecanismos de flexibilización del Protocolo de Kioto, se hipotetizaron cuáles serían las etapas que presentaría el mecanismo constitutivo esperado, subyacente a la localización de dicha

\footnotetext{
${ }^{10}$ Se entiende al término instrumentos económicos en un sentido limitado; es decir, como la adopción de medidas de regulación ambiental que permiten a los agentes contaminantes, sean estos empresas, individuos, organizaciones o Estados, elegir entre degradar el ambiente, pagando un precio por ello; o no hacerlo, y recibir una recompensa económica. Bajo esta definición, algunos de los instrumentos económicos son los impuestos y subsidios (instrumentos de precio) y los mercados de carbono o Cap and Trade (instrumentos cuantitativos) (Girardin, 2013).

${ }^{11}$ El establecimiento de un mercado de créditos de emisión debe crear incentivos a través del sistema de precios para compradores, vendedores e inversores, siempre y cuando los costes de abatimiento varíen entre países (Bernstein, 2001).
} 
norma, en el contexto doméstico brasilero. Gráficamente, este mecanismo puede representarse del siguiente modo:

\section{Mecanismo hipotetizado de los Mecanismos de Flexibilidad}

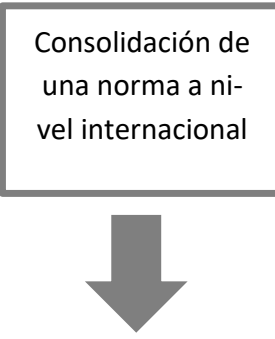

$$
\begin{aligned}
& \text { Modificación de políticas esta- } \\
& \text { tales en la materia, creación } \\
& \text { de planes y/o programas espe- } \\
& \text { cíficos al respecto, (re)asigna- } \\
& \text { ciones presupuestarias, etc. }
\end{aligned}
$$

Sanción de leyes nacionales que regulen sobre la materia de la norma

\begin{tabular}{|c|}
\hline Emergencia \\
de un número \\
creciente de \\
grupos \\
sociales \\
organizados \\
presionando \\
para un \\
cambio \\
político \\
consistente \\
con los \\
postulados de \\
la norma \\
internacional \\
\hline
\end{tabular}

\begin{tabular}{c} 
Emergencia \\
de un número \\
creciente de \\
grupos de \\
trabajo \\
gubernament \\
ales \\
formulando \\
opciones de \\
política \\
consistentes \\
con los \\
postulados de \\
la norma \\
internacional \\
\hline
\end{tabular}

\begin{tabular}{|c|}
\hline Demandas \\
para un \\
cambio en la \\
agenda \\
política \\
nacional \\
invocando la \\
norma \\
internacional \\
(especialment \\
e por parte de \\
actores no \\
estatales) \\
\end{tabular}

Fuente: Elaboración propia

Este artículo se inscribe en un programa de investigación sobre el análisis de la política climática brasileña y sus vínculos con la gobernanza global del clima, para lo cual se ha realizado un extenso trabajo de campo basado en numerosas ${ }^{12}$ entrevistas semiestructuradas a actores estatales y no estatales de Brasil. Las entrevistas fueron conducidas durante el año 2016 principalmente, tanto en forma presencial como a través de videoconferencia, mediante un muestreo no probabilístico por bola de nieve. Igualmente, se recurrió a la revisión

\footnotetext{
${ }^{12}$ Fueron realizadas 25 entrevistas en el marco más amplio de esta investigación.
} 
de quince entrevistas realizadas por Asher Lessels, quien desinteresadamente facilitó el acceso a algunas de las entrevistas que realizó en el marco de su investigación para obtener su título de maestría. Estas entrevistas fueron conducidas en forma presencial y telefónicamente con funcionarios del gobierno de Brasil del Ministerio de Ambiente, del Ministerio de Ciencia y Tecnología y del Ministerio de Relaciones Exteriores durante 2011. El anonimato se mantiene en las entrevistas citadas por un acuerdo entre los entrevistados y el autor de las entrevistas. Por otro lado, se realizó una revisión completa y sistemática de fuentes secundarias que incluyen información documental producida por actores estatales y no estatales de Brasil, así como leyes, discursos públicos, declaraciones y artículos periodísticos, entre otros. El objetivo de esta revisión ha sido evaluar la prominencia de los mecanismos de flexibilización en el contexto doméstico brasilero a partir del análisis de cada una de las etapas previstas en el proceso de localización.

Por último, cabe destacar que en los últimos años se ha avanzado en una agenda de investigación de estudios de la política climática brasileña que han sido de gran relevancia para el encuadre y el análisis del caso. Entre estos antecedentes deben ser destacados los trabajos de Albuquerque (2018, 2019), Bratman (2015), Johnson (2001), Franchini \& Viola (2019), Friberg (2009), Viola (2010), Viola \& Franchini (2011, 2012, 2014, 2018), Viola, Franchini, \& Lemos Ribeiro (2012), Viola \& Basso (2015), Vieira (2012), Vieira \& Cader (2013), Lessels (2013), Torres, Besserman, Vianna, \& Currey (2014), entre otros.

\section{Resultados}

En el contexto de consolidación del AL a nivel global, los diferentes gobiernos brasileños a partir de 1992 adoptaron rápidamente visiones pragmáticas sobre el potencial de los instrumentos económicos como herramientas de política ambiental. En este contexto, el apoyo a estos instrumentos por parte del Estado brasileño, y especialmente del Ministerio de Relaciones Exteriores (MRE de aquí en adelante), se internalizó bajo un enmarcado que los resignificaba como herramientas para obtener financiamiento externo para la promoción del desarrollo. De hecho, la aceptación de instrumentos económicos estaba atada al cumplimiento de dos condiciones:

1) En primer lugar, que la adopción de dichos instrumentos permitiese la obtención de financiamiento internacional, ya sea para el Estado brasileño, como para los estados subnacionales, y las personas físicas o jurídicas del Brasil.

2) En segundo lugar, que la adopción de dichos instrumentos no constriña la autonomía del Estado brasileño en la definición de su política doméstica y exterior.

Como resultado, la adopción de instrumentos económicos, a lo largo del período analizado, implicó que ellos fueran adoptados en el contexto y como resultado de negociaciones multilaterales, e implementados localmente a través de mecanismos que involucraban a actores del Norte global, tanto públicos como privados, como proveedores del financiamiento. Este proceso de enmarcado (framing) limitó la implementación de instrumentos económicos exclusivamente a los flujos de financiamiento internacional, dejando por fuera su uso como herramientas de política ambiental doméstica, tal como muestra la falta de implementación de un mercado de carbono (previsto originalmente en la ley brasilera de Cambio 
Climático del año 2009). Asimismo, esta interpretación habilitó a que el MRE de Brasil adquiriera un papel significativo en la definición de la política ambiental y en la adopción de dichos instrumentos.

A este respecto, en relación con la historia de los mecanismos de flexibilización, el propio gobierno de Brasil fue uno de los impulsores del Fondo para el Desarrollo Limpio (FDL), instrumento que luego se reconvertiría en el MDL. Con la entrada en vigor del PK en 2005, Brasil comienza a promover y participar activamente en la recepción e implementación de proyectos en el marco del MDL. De esta manera, cabe destacar que Brasil no solamente estuvo detrás de la idea original del MDL, sino también que, desde los inicios del mecanismo, el gobierno creó la infraestructura institucional necesaria para su funcionamiento, lo que permitió que Brasil cuente con los primeros proyectos MDL aprobados a nivel internacional ${ }^{13}$.

Para la implementación nacional de dichos proyectos, cada Estado debía establecer en su arquitectura institucional la figura de la Autoridad Nacional Designada (ADN). La ADN evalúa si el proyecto de MDL propuesto cumple con los diferentes criterios establecidos como la adicionalidad ${ }^{14}$, y si el proyecto contribuirá al desarrollo sustentable. Tal como se establecía en los Acuerdos de Marrakech ${ }^{15}$, es competencia del país anfitrión decidir qué es lo que constituye una contribución al desarrollo sustentable. Esta definición y enmarcado de los proyectos es clave para el análisis, ya que permite comprender la direccionalidad que cada país le da al Mecanismo. En Brasil, la agencia responsable de la evaluación de los proyectos era la Comisión Interministerial del Cambio Climático Global (CIMGC). Esta institución evaluaba los proyectos de acuerdo con cinco criterios básicos que fueron desarrollados en colaboración con CentroClima, un instituto de investigación de la COPPE (Escuela de Postgrado Alberto Luis Coimbra e Investigación en Ingeniería), perteneciente a la Universidad Federal de Río de Janeiro (COPPE-UFRJ). Los criterios acordados que guiaban la evaluación de proyectos eran los siguientes:

- La distribución del ingreso,

- Sostenibilidad ambiental local,

\footnotetext{
${ }^{13}$ La Junta Ejecutiva del MDL de la Secretaría de la CMNUCC aprobó como una de sus primeras iniciativas la metodología de un proyecto brasilero (Landfill-Salvador de Bahía). Del mismo modo, un proyecto brasileño (Nova Gerar), fue el primer proyecto MDL registrado por la Secretaría de la Convención Marco.

${ }^{14} \mathrm{El}$ concepto de adicionalidad es central en los proyectos de captura de carbono, tanto en términos de adicionalidad económica como ambiental. El concepto de "adicionalidad económica" plantea, esencialmente, la siguiente pregunta: ¿un proyecto se hubiera llevado a cabo de todas formas, incluso en la ausencia de los ingresos provenientes de la venta de créditos de carbono? Por su parte, el concepto de "adicionalidad ambiental" implica que el proyecto en cuestión reduce emisiones de GEI por encima de lo que hubiera ocurrido en ausencia del mismo. La adicionalidad es un elemento necesario para asegurar la integridad de cualquier reducción de emisiones obtenida por debajo de una línea de base.

${ }^{15}$ Los Ilamados Acuerdos de Marrakech refieren a las declaraciones producto de la COP VII llevada a cabo en 2001 en Marrakech, Marruecos que definieron las modalidades bajo las cuáles serían implementados los mecanismos de flexibilización definidos en 1997 con la firma del Protocolo de Kioto.
} 
- Desarrollo de condiciones de trabajo y generación neta de empleo,

- Creación de capacidad y desarrollo tecnológico,

- Integración regional e interacción con otros sectores (Friberg, 2009).

Vale la pena indicar que, como destacaran algunos entrevistados, la ADN de Brasil no se definía como promotora del $\mathrm{MDL}$, sino enmarcaba su rol en torno a asegurar la integridad ambiental del sistema; contrariamente a otras ADN, como las de China e India.

Asimismo, otro aspecto a destacar fue la creación de un procedimiento de consulta pública, transparencia e información creado por la ADN brasileña. Este procedimiento suponía que los proponentes del proyecto debían informar a las instituciones y representantes locales de la sociedad civil de su iniciativa y buscar su consentimiento. Como seguimiento de esta actividad, los desarrolladores de proyectos tenían que enviar por escrito a estos actores información sobre los principales aspectos de la implementación y operación del proyecto propuesto. Además, la regulación estipulaba que el Foro Brasileño de ONGs y Movimientos Sociales para el Medio Ambiente y el Desarrollo (FBOMS) debía ser informado sobre los proyectos propuestos, junto con al menos las siguientes instituciones clave a nivel local donde el proyecto fuera a ser implementado:

- La cámara o asociación local que nuclea y representa profesionalmente a los abogados y fiscales,

- Las autoridades municipales, tanto el poder ejecutivo como el poder legislativo local,

- Las autoridades ambientales estatales y municipales,

- Asociaciones de comunidades locales (Friberg, 2009).

Desde su creación en 2005, la ADN brasileña quedó ubicada bajo la jurisdicción del MCT y presidida por José Miguez en las labores de coordinación interministerial, como Secretario Ejecutivo del CIMGC. A partir de la creación de esta nueva institucionalidad, se definió que el secretario ejecutivo del Foro Brasileño del Cambio Climático tenía permitido asistir las reuniones del CIGMC como observador, siempre que fuera invitado por el presidente de la comisión. Las deliberaciones de este foro tenían un carácter restringido a sus miembros, no eran públicas y en general con el transcurrir de los años tendieron a hacerse cada vez más técnicas: “...la comisión interministerial se volvió cada vez más técnica, de menor nivel político y con menos poder... Después del vigor del Protocolo de Kioto, (la comisión) podía llamarse comisión de apreciación de los proyectos de MDL en Brasil." ${ }^{16}$

En relación con los sectores en los cuales se desarrollaron principalmente los proyectos MDL, hacia el año 2009 la energía renovable representaba casi la mitad de todos los proyectos MDL desplegados en Brasil. Otra parte significativa de los proyectos estaba compuesta por proyectos de captura de metano, tanto de manejo de estiércol porcino como de rellenos sanitarios (Friberg, 2009).

\footnotetext{
${ }^{16}$ Fragmento de la entrevista realizada por Asher Lessels el 14 de octubre de 2011 a un funcionario de alto rango del Ministerio de Medio Ambiente de Brasil responsable de la conducción de la delegación brasilera en la COP 9.
} 
El MDL fue valorado como un éxito por el gobierno brasileño durante los primeros años de su aplicación hasta su crisis a nivel global. Dentro de sus logros podría destacarse que el MDL alcanzó a fomentar mayor capacidad de generación de electricidad adicional que la lograda a través del programa público de promoción de energía renovable del gobierno, y los créditos de carbono también generaron un flujo de ingresos equivalente a la vigésima categoría más grande de ingresos de exportación de Brasil en el período 2005-2009 (Friberg, 2009). De esta forma, los principales méritos reconocidos por el gobierno brasileño en relación con los mecanismos MDL se asociaban al encuadre antes referido que resaltaba la obtención de financiamiento externo.

En términos de la política externa brasileña asociada a MDL, el gobierno brasileño defendía la continuación del MDL en las negociaciones post-Kioto, aunque estableciendo restricciones en la definición y delimitación de los sectores legítimos y válidos a través de los cuáles podían ser generados créditos de carbono. Así, Brasil se manifestaba en contra de permitir proyectos de captura y almacenamiento de carbono (CCS) como una categoría MDL. Esta posición se encontraba muy influenciada por la propia posición personal sostenida por José Miguez ${ }^{17}$ en el asunto, al sostener que el MDL forestal no era un mecanismo adecuado para hacer frente a las complejidades de CCS, como la responsabilidad del almacenamiento a largo plazo, y que su inclusión evitaría el desarrollo de energía renovable y crearía un incentivo positivo para la producción de combustibles fósiles, contrario al objetivo final de la convención climática. En líneas generales, Miguez compartía una posición común con la Unión Europea que temía la potencial reversión de las ganancias en términos de captura de carbono por parte de las florestas y, por ende, se oponía tanto al MDL forestal como a las metas de mitigación que se basaran en la protección forestal: "El problema es el bosque que toma carbono de la atmósfera... mantener el bosque no reduce la concentración de carbono en la atmósfera. Es un problema de la ciencia comprender si el bosque está haciendo algo o no. En el Amazonas, no hay capacidad para mantener el bosque."18

Así, el MDL forestal no logró despegar en Brasil, ya que, a pesar de las iniciativas de la sociedad civil, hacia finales del año 2009 , solo dos de los proyectos de MDL brasileños aprobados se encontraban en la categoría de forestación o reforestación, ninguno de los cuales fue finalmente aprobado por la junta ejecutiva del MDL (Friberg, 2009). Por otro lado, el rechazo de los créditos del MDL por parte del sistema de comercio de emisiones de la Unión Europea (ETS) generó una baja demanda de estos créditos sumados a los costos de transacción significativos para establecer un proyecto de forestación.

A partir de este análisis general y retomando las etapas de la hipótesis planteada en la sección anterior, para observar la localización de la norma se analizarán las distintas etapas hipotetizadas para evaluar la evidencia empírica disponible que permita sostener el argumento presentado sobre la localización de los mecanismos de flexibilización en el discurso

\footnotetext{
${ }^{17}$ Otra evidencia sobre esto se encuentra en el hecho que José Miguez se desempeñó también profesionalmente en Petrobras desde el año 2007 en adelante, y que Petrobras también se encontraba en contra de CCS en el Mecanismo para el Desarrollo Limpio, lo que era un punto de vista muy impopular entre sus pares de compañías petroleras (Friberg, 2009)

${ }^{18}$ Fragmento de la entrevista realizada por Asher Lessels el 10 de octubre de 2011 a José Miguez en Brasilia.
} 
público brasilero sobre cambio climático.

\section{i. Primera etapa}

En primer lugar, deberían encontrarse pruebas en relación con la emergencia de un número creciente de grupos sociales organizados que presionan para un cambio político consistente con los postulados de la norma internacional, así como o en forma complementaria con la emergencia de un número creciente de grupos de trabajo gubernamentales que formulen opciones de política coherentes con los postulados de la norma internacional. En este sentido, Brasil tenía una posición favorable a la adopción de mecanismos de flexibilización, ya que entendía que su adopción podía representar oportunidades de financiamiento. La adopción temprana del MDL en Brasil muestra el interés por parte del Estado en este instrumento. Cabe recuperar aquí la idea de que el MDL opera en todo el mundo con base en el mismo conjunto de reglas establecidas en los Acuerdos de Marrakech y en las decisiones posteriores de la junta ejecutiva del MDL. A pesar de esta característica común, la gobernanza del MDL varía claramente de un país de acogida a otro. Parte de esto puede remontarse a cómo estas reglas se han transpuesto e interpretado de manera diferente, así como a la diferencia en contextos y tradiciones contextuales locales.

Para describir la gobernanza del MDL, aparte del rol desempeñado por el CIMGC debe recuperarse el rol que desempeñan numerosos actores que provienen del sector privado. En primer lugar, los desarrolladores de proyectos de MDL actúan como impulsores al identificar proyectos potenciales y desarrollar ideas en proyectos. Tal como describe Friberg (2009), varias de las consultorías de MDL más exitosas que tenían presencia global fueron fundadas por brasileños e incluso si desde su inicio trasladaron su sede a otro país, mantenían presencia y actividad en Brasil. Unas pocas empresas "eco" dominaban hacia 2009 el mercado local de carbono: Ecoinvest Carbon, Econergy, MGM International, Ecosecurities, AgCert y Ecologica (Friberg, 2009). Estas firmas manejaban la mayoría de los proyectos en Brasil. En 2008, el mercado de MDL en Brasil se estaba desarrollando rápidamente con la emergencia de unas quince nuevas empresas entre aquellas que comenzaron a desarrollar proyectos y otras que se encontraban interesadas en ingresar a este nicho de mercado. Sin embargo, luego de la crisis financiera del 2008-2009, algunos de los grandes jugadores del mercado del carbono se tambalearon y se vieron obligados a cancelar partes sustanciales de sus activos (Friberg, 2009).

Por otra parte, otro actor relevante de la gobernanza del MDL eran las Entidades Operacionales Designadas (EOD). Las EODs eran auditores independientes acreditados por la Junta Ejecutiva del MDL para validar las propuestas de proyectos o verificar si los proyectos implementados han logrado las reducciones de emisiones de gases de efecto invernadero planificadas. En sus inicios, el mercado brasileño para los servicios EOD era un oligopolio dominado por tres empresas europeas. Estos actores fueron decisivos en la internalización de mecanismos de MDL en Brasil, en la medida que su grado de desarrollo y penetración no depende exclusivamente de la voluntad del sector público. Como se ha visto, en Brasil la penetración de los mecanismos MDL fue rápida en sus inicios e involucró a una pluralidad de actores en el sector privado. Para complementar la internalización del MDL en Brasil, las cámaras empresariales desempeñaron un papel importante de difusión a través de programas de capacitación para 
las empresas que estuvieran interesadas en las oportunidades económicas que se abrirían con el MDL y con el comercio de emisiones. Así, aparte de los actores empresariales vinculados a los proyectos, hubo una serie de institutos, instituciones académicas, redes internacionales y coaliciones empresariales, enre otros, que brindaron capacitaciones en la temática y brindaron apoyos a empresas que deseaban participar en dichos proyectos, así como iniciativas y proyectos lanzados en forma conjunta por actores de la sociedad civil y del sector privado en Brasil, lo que se desprende de las siguientes entrevistas: “... la CNI ya hacía los llamados cursos de inventario de emisiones, y también daba cursos de MDL. Ella hizo allí una serie de cursos, de publicaciones mostrando cómo funcionaba, cuando eso tenía una demanda muy grande por el empresariado brasileño..." ${ }^{19}$ y "Nosotros teníamos aquí una iniciativa para llevar esto al conocimiento de las empresas, para potenciar a aquellos que tuvieran interés, e incluso hicimos algunos eventos de conocimiento, de capacitación misma, sobre cómo era ese mercado, cómo esos proyectos podrían ser formulados, qué tipo de proyectos... entonces nosotros tuvimos en el pasado aquí una iniciativa, justamente para informar, qué eran esos proyectos, qué tipo de proyectos podrían presentarse, y allí hicimos ese trabajo. Sin embargo, el MDL no prosperó y allí nos detuvimos"20.

Tal como se indica en la última cita, hacia el año 2006 la FIESP organizó una serie de seminarios para capacitar al sector empresarial sobre que eran los mecanismos MDL: "Algunos sectores... lo hicimos con los sindicatos, el primero fue hecho en el interior del Estado de São Paulo, en Birigui, y tuvo participación del ayuntamiento, de todos los asociados a los sindicatos locales, una buena participación... El otro que hemos hecho, porque hemos hecho dos, fue hecho con la universidad en la región de Campinas y también tuvo una buena participación de empresas, más también de otros interesados en el tema, consultores, empleados..."21

Igualmente, varias instituciones académicas colaboraron en este proceso de difusión a través de brindar cursos destinados a ejecutivos, mandos medios y gerentes, sobre las oportunidades que podían derivar de los mercados de carbono y del MDL. Por ejemplo, la Fundación Getulio Vargas (FGV) en San Pablo y la Universidad Católica de Río de Janeiro ofrecieron cursos para sus estudiantes de MBA desde el 2005 hasta el 2010 aproximadamente sobre cómo crear y administrar proyectos de MDL. En ese proceso resultó también bastante común que el personal de estas universidades asumiera tareas de consultoría desarrollando documentos de diseño de proyectos para proyectos de MDL lateralmente a las labores específicamente académicas.

\section{ii. Segunda etapa}

Así, a partir de la consolidación de nuevos actores, comienzan a consolidarse demandas para un cambio en la agenda política nacional invocando la norma internacional. Sin embargo, dicho proceso no fue protagonizado directamente por actores no estatales, sino

\footnotetext{
${ }^{19}$ Fragmento de la entrevista realizada a Marcos Vinicius Cantarino por videoconferencia el 09 de septiembre de 2016.

${ }^{20}$ Fragmento de la entrevista realizada a Anícia Pio en San Pablo el 29 de septiembre de 2016.

${ }^{21}$ Ibidem
} 
que actores estatales tuvieron un papel muy importante también.

En este sentido, el Plan Nacional de Cambio Climático del año 2007 refiere al MDL en varias ocasiones, siendo éste un instrumento importante para asegurar el cumplimiento de los objetivos del plan. El propio plan caracteriza al MDL de la siguiente forma:

El MDL es el principal instrumento económico existente para la promoción de medidas voluntarias de mitigación de emisiones de GEI. El éxito de este instrumento en Brasil y el potencial aún existente para la implementación de nuevas actividades de proyecto en el ámbito del MDL merecen destaque. En ese sentido, el Plan Nacional preservará la adicionalidad de actividades de proyecto de MDL en Brasil, considerando que éste es un efectivo instrumento económico de promoción de acciones de mitigación de las emisiones de gases de efecto invernadero en el país. ${ }^{22}$

En el documento se destaca el rol del MDL como instrumento económico que brinda instrumentos positivos al sector privado para una mudanza de comportamiento; pero al mismo tiempo, se destaca la necesidad del gobierno de brindar reglas y normas que habiliten una institucionalización del mecanismo para aprovechar sus ventajas:

En el marco de la Convención, Brasil es responsable de numerosas iniciativas importantes, tales como la propuesta que originó el Mecanismo de Desarrollo Limpio (MDL) y el modelo para ofrecer incentivos positivos para la reducción de emisiones de deforestación en países en desarrollo... aún falta a nivel nacional un enfoque consolidado que pueda dar a las autoridades sectoriales una visión global de lo que hay detrás del potencial de las iniciativas descoordinadas de agentes privados para replicar o no actividades de proyecto MDL del sector energético, ya desarrollados en el mismo sector en otros países... ${ }^{23}$

Este discurso evidencia la internalización del principio en el contexto doméstico de Brasil, al sostener el enmarcado bajo el cual los instrumentos económicos fueron internalizados en Brasil, esto es, como mecanismos para favorecer la obtención de financiamiento internacional. "EI MDL es una oportunidad para que las empresas brasileñas para desarrollar proyectos de reducción de emisiones, por ejemplo, por el uso de energías renovables o el aumento de la eficiencia energética. En la implementación de estos proyectos todavía hay posibilidad de transferencia de tecnología y de recursos externos de empresas de países del Anexo I interesadas en obtener reducciones certificadas de emisión". ${ }^{24}$

Por otra parte, en términos de sectores, el Plan Nacional identifica que los proyectos MDL podían ser implementados en varios sectores que ofrecían oportunidades de mitigación,

\footnotetext{
22 Plan Nacional de Cambio Climático. Gobierno de Brasil. 2007.

${ }^{23}$ Ibidem

${ }^{24}$ Ibidem
} 
tales como residuos y energía. Es en este último donde son identificadas grandes oportunidades de mitigación, especialmente en el especialmente en el campo de la energía eléctrica:

Así, el MDL ayudará al país en el establecimiento de medidas adicionales de mitigación del cambio climático, conforme a la presente propuesta, sin crear cargas sustanciales a los desarrolladores del proyecto, en la medida que los créditos (las RCE) de las actividades adicionales de proyectos en el ámbito del MDL podrán ser adquiridos por países desarrollados para el cumplimiento de sus obligaciones en el marco del Protocolo de Kioto. Se constituye así una forma de minimizar la contribución brasileña para la generación de gases de efecto invernadero proveniente de la generación de energía termoeléctrica basada en combustibles fósiles. ${ }^{25}$

Según los ámbitos sectoriales definidos por el Consejo Ejecutivo del MDL, el $49 \%$ de las actividades de proyecto MDL sometidas en Brasil son de la industria de energía e involucran directamente la generación de energía eléctrica por fuente renovable. Sin embargo, todavía hay en Brasil un gran potencial inexplorado de otras actividades de proyecto MDL relacionadas con el sector de la energía. Se estima que el $67 \%$ de las metodologías aprobadas relacionadas al sector energético no se han utilizado todavía en Brasil, y el $24 \%$ sólo en una sola vez. ${ }^{26}$

Sin embargo, en sintonía con la visión prevaleciente en el Ministerio de Medio Ambiente de Brasil, el plan refiere y menciona explícitamente la posibilidad de desarrollo de programas de MDL forestal: "Se realizarán esfuerzos para el ordenamiento de estas acciones y movilizados instrumentos que contribuyan al logro de este objetivo, inclusive por medio de actividades de reforestación y forestación implementadas en Brasil en el marco del MDL, preservando la adicionalidad de este importante instrumento económico. Además, es necesario el aumento tanto de las líneas de crédito adecuadas a la actividad forestal, en cuanto a la asistencia técnica y la investigación tecnológica."

Por otro lado, en forma coherente con el desarrollo y profundización de la norma internacional, algunos actores del sector empresarial acompañados por una fracción de organizaciones de la sociedad civil impulsaron, en vinculación con actores internacionales, el desarrollo del mercado voluntario en Brasil hacia el año 2006, cuando comenzaron a operar las primeras certificadoras en Brasil a través de los ciclos de validación y verificación de los proyectos. El mercado voluntario del carbono consiste en el desarrollo de iniciativas voluntarias de reducción de emisiones a través de financiamiento proveniente de diversas fuentes. Dicha reducción de emisiones de GEls es certificada por organizaciones que respaldan que dichos proyectos reporten un beneficio ambiental. Así, en lugar de someterse a la aprobación nacional de los participantes del proyecto y al proceso de registro y verificación de la CMNUCC, el cálculo y la certificación de la reducción de emisiones se implementan de

\footnotetext{
${ }^{25}$ Plan Nacional de Cambio Climático. Gobierno de Brasil. 2007.

${ }^{26}$ Ibidem
} 
acuerdo con una serie de estándares creados por la industria. Entonces, en un mercado voluntario, quien está comprando voluntariamente está más interesado en la certificación adicional de los cobeneficios ambientales y en el compromiso con la sostenibilidad, que simplemente en los créditos derivados de la reducción de las emisiones como en el caso del MDL. A nivel global, el desarrollo de los mercados voluntarios de carbono ha sido más reducido que los mercados regulados a través de la CMNUCC, aunque han atraído bastante interés para actores de la sociedad civil, convirtiéndose en un espacio de cooperación y colaboración entre empresas y organizaciones no gubernamentales.

El desarrollo de los mecanismos de MDL por parte del sector privado y del tercer sector fue acompañado por una apuesta del gobierno brasileño en su diseño e implementación. Una vez que el MCT fue perdiendo influencia en la definición de la política exterior brasileña de cambio climático (Kiessling, 2018), Miguez y su equipo fueron designados en la nueva arquitectura institucional naciente para la implementación del MDL, siendo el propio Miguez designado como jefe ejecutivo del ADN brasileño. Miguez también se desempeñó como miembro de la Junta Ejecutiva del MDL en la Convención Marco con el apoyo del G77+China, desde 2004 hasta el año 2009 inclusive. Durante esta gestión, el ADN brasileño gana una reputación positiva y sólida tanto a nivel internacional como a nivel doméstico, especialmente en contraste con otros países del Sur Global.

Igualmente, puede rastrearse evidencia sobre el surgimiento y consolidación de un número creciente de actores sociales organizados que presionaban para un cambio político coherente con el desarrollo y profundización de medidas para abordar el problema de la desforestación desde una orientación pragmática que no privilegiaba necesariamente la utilización de instrumentos económicos por sobre otras medidas, tales como políticas de comando y control, aunque tampoco las contradecían necesariamente.

\section{iii. Tercera etapa}

Por último, la internalización de los mecanismos de flexibilización como norma en el discurso público doméstico en Brasil requería cambios en el discurso nacional y gubernamental en sintonía con los postulados de la norma internacional. En relación con este punto, MDL y CE continúan recibiendo atención como instrumentos de política por parte del gobierno al definir la posibilidad de creación de un mercado nacional de carbono en Brasil a través de la Ley Nacional de Cambio Climático del año 2009. Sin embargo, dicha iniciativa no recibió gran interés para ser desarrollada por parte del sector empresarial ni por otros actores no gubernamentales. Con relación a lo anterior, la Política Nacional de Cambio Climático implementada a través de la sancionada en 2009 establecía en su artículo 6 los instrumentos para su aplicación; en relación con el financiamiento, se definía la creación de un fondo específico (Fondo Nacional sobre Cambio Climático), así como líneas de crédito y financiamiento específicas cuya recaudación dependía de aportes del Estado nacional, más podía ser completado con recursos provenientes de fuentes internacionales de financiamiento. Asimismo, este artículo internalizaba en la legislación brasileña a: "los mecanismos financieros y económicos referentes a mitigación del cambio climático y a la adaptación a los efectos del cambio climático que existan en el ámbito de la Convención Marco de las 
Naciones Unidas sobre Cambio Climático y al Protocolo de Kioto". ${ }^{27}$

Con esta formulación, quedaban incluidos en la legislación brasileña tanto los mecanismos de flexibilización estipulados por el Protocolo de Kioto como futuros instrumentos económicos y/o financieros a crearse en el marco de la CMNUCC. Basado en antecedentes como una referencia breve en el Plan Nacional del 2007, esta política pretendía sentar las bases para la implementación de un Mercado Brasilero de Reducción de Emisiones (MBRE). En los artículos 4.8, se establece que uno de los objetivos de la política es estimular el desarrollo del MRBE. Más explícitamente, el artículo 9 de la ley 12187 define que: "El Mercado Brasileño de Reducción de Emisiones - MBRE será operacionalizado en bolsas de mercancías y futuros, bolsas de valores y entidades de mostrador organizado, autorizadas por la Comisión de Valores Mobiliarios - CVM, donde se dará la negociación de títulos mobiliarios representativos de emisiones de gases de efecto invernadero evitadas certificadas." ${ }^{28}$

Sin embargo, el mercado de carbono no fue aplicado debido a que la Comisión de Valores Mobiliarios (CVM), autoridad responsable de la regulación del mercado de capitales y de valores en Brasil, no avanzó en lo que se esperaba que fuera su competencia con la regulación del mercado que la ley de cambio climático de 2009 preveía. Al no definir un plazo concreto para su conformación, se priorizó el desarrollo de los planes sectoriales de la política. Igualmente, otra posible explicación sobre la falta de avance de la CVM en la instrumentación del mercado fue dada por una entrevistada frente a la pregunta por los motivos por los cuáles no se avanzó en la instrumentación del mercado: “Creo que porque no tenemos una meta de reducción de emisiones con foco en el sector industrial, en el sector privado. Mientras la meta se centra en el sector de la deforestación, no hay una presión ni del gobierno, ni social, para que ellos quieran, efectivamente, promover acciones." ${ }^{29}$

Entre los cambios hechos por la Política Nacional, en comparación con el Plan Nacional, debe destacarse que se incluye como instrumento de ejecución de esa política el establecimiento de estándares ambientales y de metas cuantificables de reducción y remoción de gases de efecto invernadero. Asimismo, otra novedad es el uso de criterios diferenciados en licitaciones públicas para favorecer productos y servicios que signifiquen mayor ahorro de energía, de agua y reducción en la emisión de los gases.

\section{Conclusiones}

En el presente trabajo se abordó la trayectoria de localización en el discurso doméstico en Brasil, de los mecanismos de flexibilización y la preferencia de instrumentos económicos por sobre otras formas de regulación, como norma de gobernanza global del cambio climá-

\footnotetext{
${ }^{27}$ Ley № 12.187, del 29 de diciembre de 2009. Disponible en línea en: http://www.planalto.gov.br/ccivil_03/_ato2007-2010/2009/lei/l12187.htm

${ }^{28}$ Ley № 12.187, del 29 de diciembre de 2009. Disponible en línea en: http://www.planalto.gov.br/ccivil_03/_ato2007-2010/2009/lei/I12187.htm

${ }^{29}$ Fragmento de la entrevista realizada a Beatriz Martins en San Pablo el 26 de septiembre de 2016.
} 
tico, que dan lugar en este proceso al involucramiento y participación de actores no estatales en la contestación, reinterpretación y construcción de la política climática en Brasil.

De esta manera, si bien es cierto que los actores protagonistas en el proceso de localización continuaron siendo los Estados nacionales (como actores del mercado global de emisiones, o en su rol de autoridad de aplicación de los MDL), otros actores como los estados subnacionales (al integrar mercados de carbono regionales), el sector privado (al presentar y tomar decisiones de inversión en proyectos $\mathrm{MDL}$ ), o el sector académico y el tercer sector (al aportar información sobre el MDL a tomadores de decisión), tuvieron un alto grado de participación, constituyéndose como actores cuyo accionar, sumado al del Estado nacional, merece ser analizado.

Resulta interesante en este contexto llamar la atención sobre el proceso de socialización de los actores en torno a esta norma, ya que, a partir del análisis expuesto, destacan los múltiples canales por los cuales ésta se desarrolló, sin limitarse sólo a las negociaciones internacionales, sino respondiendo a múltiples lógicas, esferas y niveles de gobernanza, con frecuencia superpuestos y altamente fragmentados. De esta manera, pese a que el origen de dichos mecanismos radica en iniciativas estatales (MDL como resultante de un acuerdo entre Estados Unidos, Japón y Brasil, CE como resultante de prácticas y acuerdos entre Estados Unidos y la Unión Europea), muy pronto dichos mecanismos dejaron de estar exclusivamente bajo el control de sus propulsores, al enmarcarse en torno a un complejo normativo mayor que respondía a ideas, discursos y normas que se encontraban profundamente enraizados en la propia sociedad internacional (Kiessling \& Pacheco Alonso, 2019).

Así, en este artículo, bajo la pregunta de cómo los mecanismos de flexibilización establecidos por el Protocolo de Kioto fueron internalizados en la política doméstica brasilera, se atiende a las modalidades y las condiciones bajo las cuáles se llevaron adelante los debates para la implementación del Mecanismo de Desarrollo Limpio y el Comercio de Emisiones, y las ideas bajo las cuales estos se introdujeron en la política climática brasilera. En particular, el contraste entre las etapas hipotetizadas en el proceso de convergencia normativa y localización de los mecanismos de flexibilización y la evidencia recabada denota, como se ha observado, que el proceso de construcción de convergencia normativa con relación a los mecanismos de flexibilización en Brasil no ha sido necesariamente lineal. Sin embargo, la prueba empírica más importante en términos de los esquemas presentados (la efectiva existencia de cambios en el discurso nacional y gubernamental en sintonía con los postulados de la norma internacional) se observa en el contexto político brasileño como un indicador de este proceso de localización, especialmente del MDL.

En términos más amplios, el análisis del caso brasilero muestra que la comprensión de normas internacionales como procesos, resulta importante para comprender la construcción de convergencia normativa como una dinámica dialéctica entre los cambios en la gobernanza internacional y en los cambios en el plano doméstico. Acontecimientos importantes y significativos en el plano local, así como coyunturas críticas, permiten acelerar dichas dinámicas, tal como sucedió con la sanción de la ley de cambio climático del año 2009 y la definición de una Política Nacional de Cambio Climático. Sin embargo, tal proceso de aceleración no implica ni garantiza el éxito de la implementación de la norma, en la medida que 
ciertas reticencias de algunos actores del sector privado tendieron a demorar la implementación de un mercado nacional de emisiones luego del año 2011 con un debilitamiento del impacto efectivo de estos instrumentos en la mitigación de emisiones en Brasil.

En síntesis, la presente investigación permite arribar a dos ideas centrales con respecto al caso analizado:

La introducción de los mecanismos de flexibilización en Brasil se vincula a la hegemonía del discurso asociado al ambientalismo liberal, en el marco del cual se inscriben instrumentos como el Mecanismo de Desarrollo Limpio y el Comercio de Emisiones con respecto a la gobernanza climática. El MDL incluso tuvo su origen en una iniciativa brasilera; sin embargo, en el proceso tendiente a su institucionalización a nivel internacional y su consiguiente reintroducción a la esfera doméstica brasilera, sufrió transformaciones que responden a la localización de normas internacionales.

La sociedad civil y otros actores subnacionales y no estatales adquirieron una gran relevancia en la internalización e implementación de estas normas. La evidencia denota que el Mecanismo de Desarrollo Limpio y el Comercio de Emisiones fueron internalizados principalmente a partir de diversas iniciativas y proyectos lanzados por actores de la sociedad civil y del sector privado en Brasil; lo que llevó a que, en el período comprendido entre 2005 y 2008 , Brasil fuera el tercer país receptor de proyectos MDL, detrás de China e India. Sumado a los actores empresariales vinculados a los proyectos, hubo una serie de institutos, instituciones académicas, redes internacionales y coaliciones empresariales, entre otros, que brindaron capacitaciones en la temática, y apoyaron y acompañaron a empresas que deseaban participar en dichos proyectos. Así, se observa una reestructuración tanto de los actores internos que lideran la gobernanza del cambio climático, como del espectro de temas relevantes en la regulación climática en Brasil.

\section{Bibliografía}

Abdelal, R. (2009). Constructivism as an approach to international political economy. In M. Blyth, Routledge Handbook of International Political Economy (IPE). IPE as a Global Conversation (pp. 62-76). Routledge.

Abdelal, R., Blyth, M., \& Parsons, C. (2010). Constructing the International Economy. Cornell University Press.

Acharya, A. (2004). How Ideas Spread: Whose Norms Matter? Norm Localization and Institucional Change in Asian Regionalism. International Organization, 239-275.

Acharya, A. (2011). Norm Subsidiarity and Regional Orders: Sovereignty, Regionalism, and Rule-Making in the Third World. International Studies Quarterly, 95-123.

Acharya, A. (2012). Ideas, norms, and regional orders. In P. Thazha Varkey, International Relations Theory and Regional Transformation (pp. 183-209). Cambridge University Press.

Albuquerque, F. L. (2018). Developing Powers in Multilateral Regimes: Brazil's Foreign Policy in the Climate Change and Peace and Security regimes. International 
Negotiation, 423-445.

Albuquerque, F. L. (2019). Coalition Making and Norm Shaping in Brazil's Foreign Policy in the Climate Change Regime. Global Society, 243-261.

Bernstein, S. (2001). The compromise of liberal environmentalism. Columbia University Press.

Bratman, E. Z. (2015). Brazil's Ambivalent Challenge to Global Environmental Norms. In O. Stuenke, \& M. M. Taylor, Brazil on the Global Stage. Power, Ideas, and the Liberal International Order (pp. 95-116). Palgrave Macmillan.

Franchini, M., \& Viola, E. (2019). Myths and images in global climate governance, conceptualization and the case of Brazil (1989 - 2019). Revista Brasileira de Política Internacional, 1-21.

Friberg, L. (2009). Varieties of Carbon Governance: The Clean Development Mechanism in Brazil- a Success Story Challenged. The Journal of Environment \& Development, 395-424.

Girardin, O. (2013). Aspectos Socioeconómicos y Políticos del Cambio Climático. De la Convención al Protocolo de Kioto Tomo I (1990-2000). Fundación Patagonia Tercer Milenio.

Katz-Rosene, R., \& Matthew, P. (2020). Thinking Ecologically About the Global Political Economy (RIPE Series in Global Political Economy). Routledge.

Kiessling, C. (2018). Brazil, Foreign Policy and Climate Change (1992-2005). Contexto Internacional, 387-408.

Kiessling, C. (2019). Internalización Del Principio De Las Responsabilidades Comunes, Pero Diferenciadas: Interpretaciones Desde La Sociedad Civil Brasileña. Letras Verdes. Revista Latinoamericana De Estudios Socioambientales, 8-28.

Kiessling, C. (2019). Vinculación entre la política climática doméstica en países emergentes y la política internacional del cambio climático: propuesta de un marco analítico. Comillas Journal of International Relations, 78-98.

Kiessling, C. \& Pacheco Alonso, A. (2019). Escuela inglesa y gobernanza climática global: un diálogo necesario, Relaciones Internacionales, 53-72.

La Riviere, L. (2014). Towards a Constructivist International Political Economy of Climate Change. Issues in Political Economy, 90-101.

Lessels, A. (2013). Cambiando el clima, el efecto de Lula sobre la posición de Brasil en las negociaciones de la UNFCCC. Flacso Argentina, Universidad de San Andrés, Universitat de Barcelona.

Levy, D., \& Spicer, A. (2013). Contested imaginaries and the cultural political economy of climate change. Organization, 659-678.

Ruggie, J. (1982). International regimes, transactions and change: embedded liberalism in the postwar economic order. International Organization, 379-415.

Saguier, M. (2018). El ambiente en la Economía Política Internacional. XIII Congreso Nacional 
y VI Congreso Internacional sobre Democracia. Universidad Nacional de Rosario.

Torres, E., Besserman Vianna, S., \& Currey, K. (2014). Brazil: An Emerging Economic Power Committed to Sustainability. In J. Colombano, \& A. Shah, Learning from the World. New Ideas to Redevelop America (pp. 142-164). Palgrave Macmillan.

Vieira, L., \& Cader, R. (2013). A política ambiental na década 2002-2012. In E. Sader, 10 anos de governos pós-neoliberais no brasil: Lula e Dilma (pp. 225-238). Boitmepo Editorial. Flacso Brasil.

Vieira, M. (2012). Brazilian Foreign Policy in the Context of Global Climate Norms. Foreign Policy Analysis, 1-18.

Viola, E. (2010). A política climática global e o Brasil: 2005-2010. Revista Tempo do mundo, 81-117.

Viola, E., \& Basso, L. (2015). Brazilian Energy-Climate Policy and Politics towards Low Carbon Development. Global Society, 427-446.

Viola, E., \& Franchini, M. (2011). A mudança climática em 2011: governança global estagnada e o novo perfil de emissões de Brasil. Breves cindes.

Viola, E., \& Franchini, M. (2012). Climate politics in Brazil: Public Awareness, Social Transformations and Emissions Reduction. In I. Bailey, \& H. Compston, Feeling the Heat. The politics of Climate Policy in Rapidly Industrializing Countries (pp. 175-204). Palgrave Macmillan.

Viola, E., \& Franchini, M. (2014). Brazilian climate politics 2005-2012: ambivalence and paradox. Wires Climate Change, 677-688.

Viola, E., \& Franchini, M. (2018). Brazil and Climate Change: Beyond the Amazon. Routledge.

Viola, E., Franchini, M., \& Lemos Ribeiro, T. (2012). Sistema internacional de hegemonia conservadora: Governança global e democracia na era da crise climática. Annablume.

Widmaier, W. (2003). The Keynesian Bases of a Constructivist Theory of the International Political Economy. Millennium, Journal of International Studies, 87-107.

Williamson, J. (1989). What Washington Means by Policy Reform. In J. Williamson, Latin American Readjustment: How Much has Happened. Washington: Institute for International Economics. 